\title{
Hydrolytic Degradation of Poly(L-Lactide-co-Glycolide) Studied by Positron Annihilation Lifetime Spectroscopy and Other Techniques
}

\author{
E. Pamula ${ }^{a, *}$, E. DryzeK $^{b}$ And P. Dobrzyński $^{c}$ \\ ${ }^{a}$ Faculty of Materials Science and Ceramics, Department of Biomaterials \\ AGH University of Science and Technology \\ al. Mickiewicza 30, 30-059 Kraków, Poland \\ ${ }^{b}$ Institute of Nuclear Physics, Radzikowskiego 152, 31-342 Kraków, Poland \\ ${ }^{c}$ Centre of Polymer Chemistry, Polish Academy of Sciences \\ Curie-Skłodowskiej 34/20, 41-819 Zabrze, Poland
}

\begin{abstract}
Changes of the poly(L-lactide-co-glycolide) structure as a function of degradation time in phosphate-buffered saline for 7 weeks were investigated by gel permeation chromatography, differential scanning calorimetry, nuclear magnetic resonance $\left({ }^{1} \mathrm{H}\right.$ NMR), and positron annihilation lifetime spectroscopy. Surface properties as wettability by sessile drop and topography by atomic force microscopy were also characterized. Chain-scission of polyester bonds in hydrolysis reaction causes a quite uniform decrease in molecular weight, and finally results in an increase in semicrystallinity. Molecular composition of the copolymer and water contact angle do not change considerably during degradation time. Atomic force microscopy studies suggest that the copolymer degrades by "in bulk" mechanism. The average size of the molecular-level free volume holes declines considerably after one week of degradation and remains constant till the sixth week of degradation. The free volume fraction decreases as a function of degradation time.
\end{abstract}

PACS numbers: 82.35.-x, 82.35.Lr, 78.70.Bj, 61.18.Fs, 82.56.Ub, 83.85.Jn, 68.37.Ps

\section{Introduction}

Degradable aliphatic polyesters: polylactides (PLLA, PDLA, PDLLA), polyglycolide (PGA) and their copolymers (PLG) are widely used in medicine (sutures, internal fracture fixation devices), pharmacy (drug delivery systems) and

*corresponding author; e-mail: epamula@agh.edu.pl 
tissue engineering (scaffolds acting as artificial extra-cellular matrix) [1-3]. These polymers undergo degradation in aqueous media by chain-scission of polyester bonds in hydrolysis reaction. Their degradation products (lactic acid and glycolic acid) are removed from the body by normal metabolic pathways [3].

Hydrolytic degradation of aliphatic polyesters has been the subject of many studies over the last two decades [4-7]. It was shown that the mechanism of hydrolytic degradation is quite unusual, because very often degradation is heterogeneous: faster inside the sample than at their surface $[4,5]$. Degradation kinetics depends on many factors such as chemical and chain structure, crystallinity, molecular mass and their polydispersion, presence of low-molecular weight oligomers, as well as properties of the aqueous medium like $\mathrm{pH}$, ionic strength, temperature, and buffering capacity $[1,3-5]$.

Positron annihilation lifetime spectroscopy (PALS) is a technique well established in polymer characterization and it is often used to investigate free volume holes $[8,9]$. Positron injected into the polymer form the positron-electron bound state called positronium (Ps). The positronium is trapped in the regions of low electron density which are free volume holes of the radius in the range from 0.2 to $0.6 \mathrm{~nm}$. The positronium has two spin states para-Ps ( $p$-Ps) and ortho-Ps $(o-\mathrm{Ps})$. In molecular solids $o$-Ps localized in the free volume hole annihilates with an electron of opposite spin from molecules forming free volume hole walls. This process is called pick-off annihilation. The analysis of the PALS spectra of the polymers gives usually three lifetime components: $\tau_{1}$ attributed to $p$-Ps annihilation, $\tau_{2}$ attributed to free positron annihilation and $\tau_{3}$ attributed to $o$-Ps annihilation in the pick-off process. Each lifetime has a corresponding intensity related to the number of annihilations with a particular lifetime. The mean free volume hole radius is related to $\tau_{3}$. As the free volume holes become smaller, the local electron density seen by $o$-Ps increases and its lifetime value decreases which was proven experimentally and theoretically $[8,9]$.

Ps probes the free volume holes in the polymer structure located between chains and at chains ends. The size of the holes corresponds also to molecular radii of diffusing substances (oxygen, nitrogen, carbon dioxide, water); therefore gas transport properties of polymeric materials (e.g. permeability and diffusivity) are often correlated with the size of free volumes and free volume fraction [10-13]. Moreover, PALS was applied to study the structure of different polymers after $\gamma$ irradiation [14], thermal [15] and outdoor degradation [16], because usually degradation influences the size and distribution of molecular level free volume holes. So far, PALS has not been used to analyze structural changes in aliphatic polyesters caused by hydrolytic degradation.

In this paper we attempt to find a relationship between structure and properties of poly(L-lactide-co-glycolide) (PLG) as a function of incubation time in aqueous medium (phosphate-buffered saline, PBS) in order to better understand degradation of aliphatic polyesters. 


\section{Experimental}

\subsection{Materials}

Glycolide and L-lactide (Purac, The Netherlands) were purified by recrystallization from dry ethyl acetate in a vacuum dryer at a room temperature. Copolymerization of glycolide and lactide was performed in bulk with a $\mathrm{Zr}$ (acac) $)_{4}$ initiator at $100^{\circ} \mathrm{C}$ by a conventional method using a vacuum line for degassing and sealing of the ampoules as described previously [17]. The molar ratio of the initiator and the monomers was $1.2 \times 10^{-3}$. In order to remove unreacted monomers, the copolymer was purified by dissolution in chloroform and precipitation in methanol followed by drying in vacuum at $50^{\circ} \mathrm{C}$ until constant weight.

Copolymer foils were made by solvent-casting from $10 \% \mathrm{w} / \mathrm{v}$ (weight per volume) copolymer solution in methylene chloride (POCH S.A., Gliwice, Poland) on glass Petri dishes followed by air and vacuum drying at a decreased pressure. The foils thickness was $0.17 \pm 0.01 \mathrm{~mm}$.

\subsection{Degradation conditions}

Degradation of copolymer foils was performed at $37^{\circ} \mathrm{C}$ in phosphate-buffered saline [137 mM NaCl, $24 \mathrm{mM} \mathrm{Na}_{2} \mathrm{HPO}_{4}$ and $16 \mathrm{mM} \mathrm{KH} \mathrm{PO}_{4}, \mathrm{pH}=7$ ]. The foils weighing $0.23 \mathrm{~g}( \pm 0.02 \mathrm{~g})$ were incubated in $100 \mathrm{ml}$ of PBS in plastic vials for 7 weeks. PBS was changed once a week. Every week one piece of foil was washed 5 times in ultra-high purity water of the resistivity of $18.2 \mathrm{M} \Omega \mathrm{cm}^{-1}$ (UHQ-water) (purified by Purelab UHQ, Elga, Germany), and dried in a vacuum oven for at least $24 \mathrm{~h}$. The relative mass change was calculated from the formula

$$
M_{\mathrm{R}}=\frac{m_{\mathrm{t}}-m_{0}}{m_{0}} 100 \%,
$$

where $m_{\mathrm{t}}$ - mass after incubation [g], $m_{0}$ - initial mass [g].

\subsection{Methods}

The copolymer composition was determined by ${ }^{1} \mathrm{H}$ NMR measurements with a Varian Unity Inowa spectrometer and a $5 \mathrm{~mm}$ sample tube. Dried dimethyl sulfoxide- $\mathrm{d}_{6}$ was used as a solvent and the spectra were obtained at $100^{\circ} \mathrm{C}$ with 32 scans, a $3.74 \mathrm{~s}$ acquisition time, and a $7 \mu$ s pulse width.

Molecular weight, $M_{n}$, and polydispersity, $d$, were determined by gel permeation chromatography with the Spectra Physics SP 8800 chromatograph (chloroform was used as the eluent; the flow rate was $1 \mathrm{~mL} / \mathrm{min}$; Styragel columns and a Shodex SE 61 detector were used).

The intrinsic viscosity of foils was measured by Ubbelohde viscometer in chloroform at $25^{\circ} \mathrm{C}$, and the intrinsic viscosity was calculated for the concentration of solution $0.8 \mathrm{~g} / \mathrm{dL}$, according to the formula [18]:

$$
\eta=\frac{\left[2\left(\frac{t}{t_{0}}-\ln \left(\frac{t}{t_{0}}\right)-1\right)\right]^{1 / 2}}{c},
$$

where $c$ - concentration of the solution, $t$ - flow time of the solution, $t_{0}$ - flow time of pure solvent. 
Thermal properties, such as glass-transition temperature $\left(T_{\mathrm{g}}\right)$, melting temperature $\left(T_{\mathrm{m}}\right)$ and heat of melting $\left(\Delta H_{\mathrm{m}}\right)$ were studied using differential scanning calorimetry (DSC) with DuPont 1090B apparatus calibrated with gallium and indium.

Topography measurements were performed under ambient laboratory conditions with an Explorer atomic force microscope (ThermoMicroscopes, Vecco, USA). Contact mode topographic images were recorded using $\mathrm{Si}_{3} \mathrm{~N}_{4}$ tips with a spring constant of $0.05 \mathrm{~N} / \mathrm{m}$ and a nominal radius of curvature of $20 \mathrm{~nm}$ (Vecco NanoProbe ${ }^{\mathrm{TM}}$ Tips, model MLCT-EXMT-A). The images were recorded at scan area $100 \mu \mathrm{m} \times 100 \mu \mathrm{m}$ for three aleatory chosen places $(300 \times 300$ data points $)$ and with scan rate of 5 lines/s. All images were flattened using a third order polynomial algorithm provided with the instrument.

The contact angle was measured by sessile drop method by an automatic drop shape analysis system DSA $10 \mathrm{Mk} 2$ (Kruss, Germany). The UHQ-water droplet was $0.2 \mu \mathrm{l}$ and each determination was obtained by averaging the results of 12 measurements.

Positron lifetime spectra were measured using a conventional fast-fast spectrometer with $\mathrm{BaF}_{2}$ scintillators. The time resolution of the system was 250 ps (FWHM). The $30 \mu \mathrm{Ci}$ activity positron source containing ${ }^{22} \mathrm{Na}$ isotope enveloped in $7 \mu \mathrm{m}$ thick kapton foil was sandwiched between two polymer samples. Lifetime spectra containing $\approx 2 \times 10^{6}$ counts were measured at room temperature. All obtained spectra were decomposed using the LT code; subtracting the background and the source component [19]. The obtained positron lifetime spectra were decomposed into three components. The first component value $\tau_{1}$ was fixed at $0.125 \mathrm{~ns}$. The value of $\tau_{2}$ was ranged from $0.37 \mathrm{~ns}$ to $0.39 \mathrm{~ns}$ for all samples. In this study we are discussing the longest-lived component, $\tau_{3}$. Average free volume was calculated according to the semiempirical model $[20,21]$ combining the pick-off lifetime $\tau_{3}$ with the average free volume radius of a spherical hole $V_{\mathrm{f}}=4 \pi R^{3} / 3$ :

$$
\tau_{3}=\frac{1}{2}\{1-R /(R+\Delta R)+(1 / 2 \pi) \sin [2 \pi R /(R+\Delta R)]\}^{-1},
$$

where $R$ is radius of the hole and $\Delta R$ is an electron layer thickness. The value of $\Delta R$ was estimated as $1.66 \AA$ by fitting the longest lifetime value to known free volume sizes of molecular crystals [8].

\section{Results}

At the beginning of the experiment poly(L-lactide-co-glycolide) foils were transparent because of their amorphous structure. After 5 weeks of incubation in PBS they became white and opaque, and after 7 weeks they started to crumble.

Figure 1 shows evolution of the number-average molecular weight and polydispersity of the copolymer foil during their incubation in PBS. $M_{n}$ decreases from about $60 \mathrm{kDa}$ before, to about $4 \mathrm{kDa}$ after a 7 -week degradation. An increase in polydispersity is detectable, but the molecular weight distribution remains monomodal. 


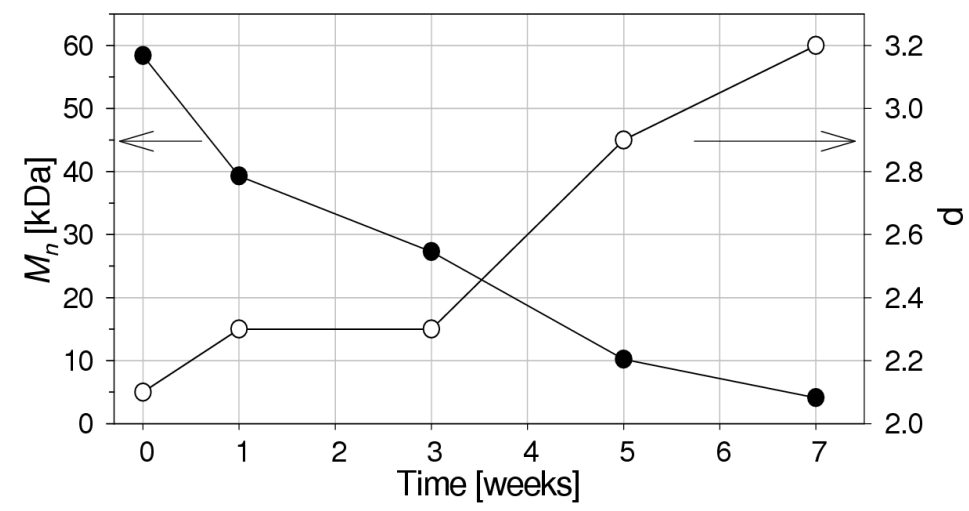

Fig. 1. Variations of number average molecular weight, $M_{n}$ (left axis), and polydispersity, $d$ (right axis), of the poly(L-lactide-co-glycolide) foils as a function of incubation time in PBS.

The results of viscosity, NMR, mass changes, DSC and contact angle are presented in Table. Incubation in PBS causes a rapid and uniform decrease in viscosity from about $1.1 \mathrm{dL} / \mathrm{g}$ before to about $0.4 \mathrm{dL} / \mathrm{g}$ after the 7 -week degradation. Chemical composition of the copolymer does not change considerably during degradation; only a slight increase in glycolide molar fraction by the third week of incubation, followed by a decrease by the seventh week are detectable. The foils lose up to $12 \%$ wt mass in 7 weeks; this is caused by random scission of polyester bonds in hydrolysis reaction, which results in leaching of water-soluble low-molecular weight oligomers to the aqueous medium.

TABLE

Properties of poly(L-lactide-co-glycolide) foils as a function of degradation in PBS. $t$ - incubation time in PBS, $\eta$ - intrinsic viscosity, $N_{\mathrm{G}}-$ molar fraction of glycolide, $M_{\mathrm{R}}$ - mass changes, $T_{\mathrm{g}}$ - glass transition temperature, $T_{\mathrm{m}}$ - melting temperature, $\Delta H_{\mathrm{m}}$ - heat of melting, $\theta$ - water contact angle, $n=12$, standard deviation in brackets.

\begin{tabular}{c|c|c|c|c|c|c|c}
\hline \hline $\begin{array}{c}t \\
\text { [weeks] }\end{array}$ & $\begin{array}{c}\eta \\
{[\mathrm{dL} / \mathrm{g}]}\end{array}$ & $\begin{array}{c}N_{\mathrm{G}} \\
{[\text { mole \%] }}\end{array}$ & $\begin{array}{c}M_{\mathrm{R}} \\
{[\%]}\end{array}$ & $\begin{array}{c}T_{\mathrm{g}} \\
{\left[{ }^{\circ} \mathrm{C}\right]}\end{array}$ & $\begin{array}{c}T_{\mathrm{m}} \\
{\left[{ }^{\circ} \mathrm{C}\right]}\end{array}$ & $\begin{array}{c}\Delta H_{\mathrm{m}} \\
{[\mathrm{J} / \mathrm{g}]}\end{array}$ & $\begin{array}{c}\theta \\
{\left[{ }^{\circ}\right]}\end{array}$ \\
\hline 0 & 1.08 & 15.1 & 0 & 57.8 & - & - & $77.2(2.1)$ \\
1 & 0.91 & 15.8 & -2.48 & 56.4 & - & - & $76.6(3.1)$ \\
3 & 0.83 & 16.2 & -3.10 & 56.6 & - & - & $77.8(4.9)$ \\
5 & 0.49 & 15.6 & -7.89 & 51.5 & 127.1 & 1.7 & $77.3(3.8)$ \\
7 & 0.44 & 15.0 & -10.43 & 50.0 & 128.2 & 16.8 & $76.1(2.7)$
\end{tabular}

A DSC thermogram of the initial copolymer foil exhibits only a small peak at $57.8^{\circ} \mathrm{C}$ originating from glass transition $\left(T_{\mathrm{g}}\right) . T_{\mathrm{g}}$ does not change its position 
up to the fourth week of degradation. $T_{\mathrm{g}}$ decreases to $51.5^{\circ} \mathrm{C}$ and $50^{\circ} \mathrm{C}$ for the samples degraded after 5 and 7 weeks, respectively, correlating with the decrease in molecular weight. After 5 weeks of degradation, a small melting peak $\left(T_{\mathrm{m}}\right)$ at $127.1^{\circ} \mathrm{C}$ is detectable. After 7 weeks, $T_{\mathrm{m}}$ shifts to $128.1^{\circ} \mathrm{C}$ and a huge increase in the melting enthalpy is observed, indicating an increase in crystallinity. Simultaneously, the samples lose their transparency. It indicates that during copolymer structure degradation, small-length molecular chains are able to organize, thus increasing the average crystallinity of the copolymer.

Incubation in PBS has no effect on water contact angle of PLG during the whole experiment, suggesting that the copolymer does not change its hydrophobicity when degrading.
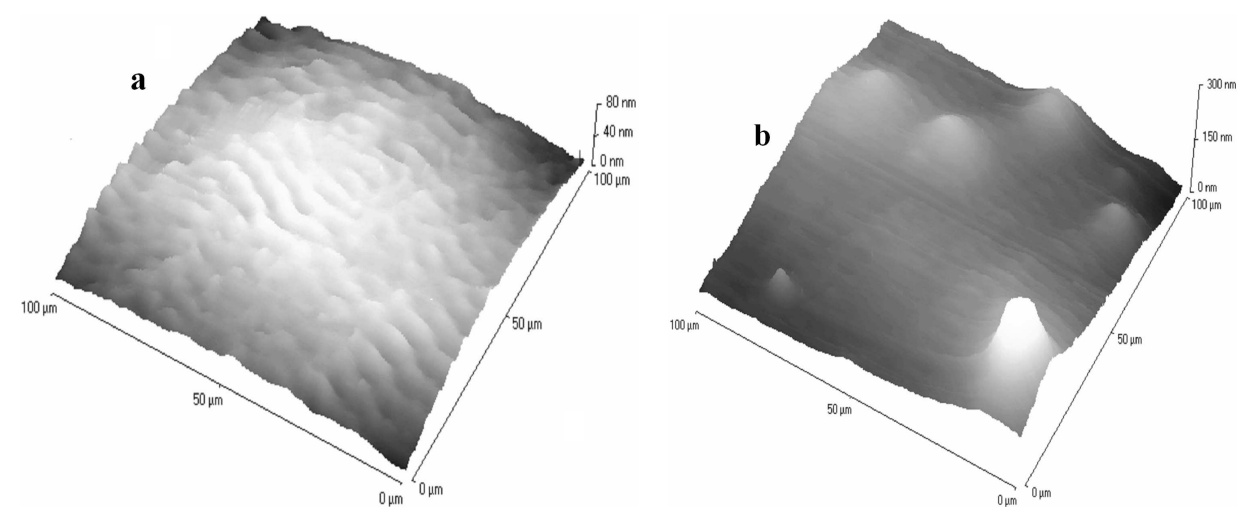

Fig. 2. AFM topographical images of poly(L-lactide-co-glycolide) surface (scan area $100 \mu \mathrm{m} \times 100 \mu \mathrm{m})$ : (a) before degradation $(z=70 \mathrm{~nm}$ ), (b) after 5 weeks in PBS $(z=300 \mathrm{~nm})$.

Figure 2 presents atomic force microscopy (AFM) topographical images of PLG surface before and after 5 weeks of incubation in PBS. Wave-like features less than $70 \mathrm{~nm}$ in height on the surface of PLG before degradation are present (Fig. 2a). Such topographical features are characteristic of polymeric foils made by slip casting and are created during solvent evaporation. Bungles of up to $300 \mathrm{~nm}$ in height were observed on the surface after incubation in PBS (Fig. 2b). The presence of a hill-like topographical structures after incubation in PBS confirms that surface erosion is not the main mechanism of PLG degradation and rather the foils degrade mainly through the "in bulk" mechanism.

Figure 3 presents the variations of the average free volume size of the copolymer foils, $V_{\mathrm{f}}$, calculated from the longest-lived component of positron spectra, $\tau_{3}$ (a), and their intensity $I_{3}$ (b) as a function of degradation time. After the first week of degradation, a significant decrease in the $\tau_{3}$ takes place whereas after the second week, $\tau_{3}$ remains almost constant. Decrease in $\tau_{3}$ coincides with a similar decrease in free volume radius, from $2.88 \pm 0.01 \AA$ for the initial sample to 


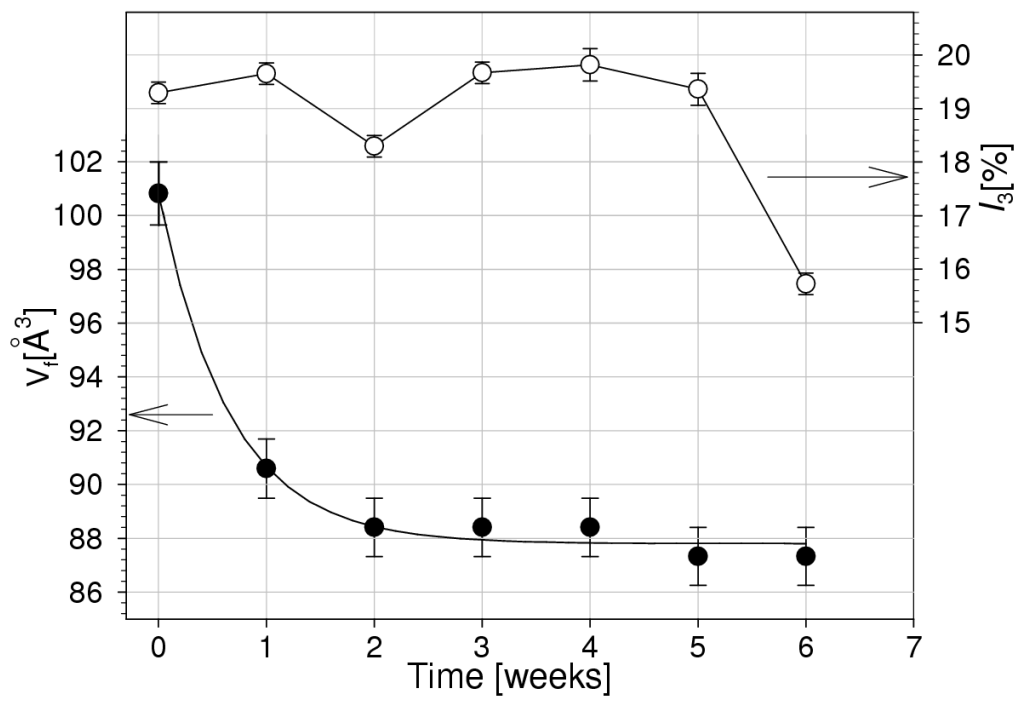

Fig. 3. Average free volume hole size $V_{\mathrm{f}}$ (left axis) and intensity $I_{3}$ (right axis) of the poly(L-lactide-co-glycolide) foils as a function of incubation time in PBS.

$2.76 \pm 0.01 \AA$ for the polymer after 6 weeks of degradation. The relationship of the average free volume size on degradation time can be described by the exponential decay function given by the equation: $V_{\mathrm{f}}=V_{\mathrm{f} 0}+A \exp (-B t)$, with the following parameters: $V_{\text {f0 }}=88.5 \pm 0.3 \AA^{3}, A=11.6 \pm 0.5 \AA^{3}, B=1.51 \pm 0.2$ week $^{-1}$. The intensity of the $o$-Ps annihilation, $I_{3}$, decreases significantly from $c a .19 .5 \%$ to $15.7 \%$ after six weeks of degradation.

\section{Discussion}

Degradation of aliphatic polyesters is very complex and depends on many factors. In this study, in vitro degradation of poly(L-lactide-co-glycolide) foils in PBS at $37^{\circ} \mathrm{C}$ was investigated and the results of mass loss, viscosity, molecular weight and its distribution, chemical structure, thermal properties, wettability, topography and parameters obtained from positron annihilation spectroscopy throughout degradation were analysed.

During the 7-week observation the PLG foils changed colour, from transparent to white, and finally became extremely brittle with negligible strength. The change of colour appeared in the samples after 5 weeks of incubation coinciding with a tremendous decrease in viscosity and molecular weight. The reduced molecular weight, due to chain-scission of polyester bonds in hydrolysis reaction can explain an increase in chain mobility, thus allowing an easier arrangement in crystalline structures. Interestingly, chemical composition did not change considerably during degradation, showing that the process of hydrolysis did not influence preferably glycolyl and lactyl segments in the copolymer structure. Wettability and topography observations show that the mechanism of degradation cannot be 
explained by simple surface erosion. In contrast, the foils underwent degradation in bulk, as observed before by others [3-6]. A considerable loss of mass indicates that the low-molecular weight compounds and soluble oligomers were released from the material during degradation.

As it follows from the PALS spectroscopy, a significant decrease in the subnanometer free volume holes size from $100 \AA^{3}$ to $90 \AA^{3}$ was observed only for samples after a 1-week degradation in PBS. The size of the molecular defects, for the samples degraded for longer periods of time, did not change considerably, although other macroscopic parameters such as molecular weight and crystallinity changed to a great extent resulting in disintegration of copolymer foil after the 7-week incubation.

Changes in the free volume size in polymer structure have been often combined with an increase in crystallinity and decrease in molecular weight [16, 22-24]. During the process of polymer degradation, a reduction of molecular weight results in increase in molecular mobility which in turn allows for an easier arrangement of shorter polymer chains in crystalline structures. Such a phenomenon accompanied by reduction of the free volume hole size was observed in poly(ethylene terephtalate) as a result of hydrolysis [22] or photodegradation [23]. Nevertheless, in the case of poly(ethylene terephtalate) $\gamma$-irradiation [24] or isotactic polypropylene environmental degradation [16], positron lifetime measurements did not show a decrease in the $o$-Ps lifetime that could explain a reduction of the free volume size. In that case a drop of the $o$-Ps intensity was well correlated with an increase in crystallinity resulting from a decreased volume of amorphous regions without shrinking the free volume holes.

In our experiment, a drop of the free volume size was observed only during the first week of degradation, while a reduction of the molecular weight took longer. Therefore, it seems that there is not a direct relationship between the size of subnanometer free volume holes in the PLG structure and macroscopic properties of the copolymer, such as molecular weight and its distribution or viscosity. Measured decrease in the free volume holes size after 1 week of degradation is presumably caused by processes connected with absorption of water molecules in the free volume holes which finally result in hydrolysis of polyester bonds.

There is a consensus in the literature that the first event after immersion of aliphatic polyesters (poly-DL-lactide, copolymers of L-lactide and glycolide) in aqueous media is water absorption [4]. The penetrating water molecules create a negative water concentration gradient from the surface to the centre of the device as would be expected through diffusion. This gradient disappears after several days and hydrolysis of ester bonds is homogeneous in the first stage. Afterwards, degradation is heterogeneous: the surface and sub-surface region degrades more slowly than inner parts of the device, due to autocatalytic effect of carboxylic chain ends created during hydrolysis and trapped inside the polymer matrix $[4,5]$. 
Therefore, PALS seems to be useful tool to probe structural changes of aliphatic polyesters during the first stage of hydrolytic degradation resulting from water uptake. Further stages of degradation provoking creation of hollow structures inside and bungles on the surfaces have no impact on the free volume size. It could be expected that chemical structure changes connected with increase in amount of oxygenated chemical groups, which inhibit Ps creation, should reduce intensity of $o$-Ps pick-off annihilation component, $I_{3}$. Decrease in $I_{3}$ is registered after six weeks of degradation unless taking into consideration a small drop of this value after the second week of degradation. On the other hand, decrease in $I_{3}$ after six weeks of degradation is well correlated with increase in polymer crystallinity, as shown by DSC.

In conclusion, copolymer of L-lactide and glycolide degrades in bulk through hydrolysis of polyester bonds, which results in decrease in molecular weight, followed by increase in crystallinity. In spite of complexity of this process at molecular level, positron annihilation spectroscopy reveals reduction in the free volume hole size in the first stage of degradation process, connected with water absorption. The increase in crystallinity of the copolymer and/or changes in chemical structure may be correlated with $o$-Ps annihilation intensity.

\section{Acknowledgments}

This study was financed from the Polish Budget Founds for Scientific Research within the years 2005-2008 (research project no. 3 T08D 019 28).

\section{References}

[1] R. Chandra, R. Rustgi, Prog. Polym. Sci. 23, 1273 (1998).

[2] S.-J. Sieh, J.P. Vacanti, Surgery 257, 1 (2005).

[3] B.L. Seal, T.C. Otero, A. Panitch, Mater. Sci. Eng. R 34, 147 (2001).

[4] I. Grizzi, H. Garreau, S. Li, M. Vert, Biomaterials 16, 305 (1995).

[5] S. Li, S. McCarthy, Biomaterials 20, 35 (1999).

[6] X. Yuan, A.F.T. Mak, K. Yao, Polym. Degrad. Stab. 75, 45 (2002).

[7] E. Pamuła, P. Dobrzyski, M. Bero, C. Paluszkiewicz, J. Mol. Struct. 744-747, 557 (2005).

[8] Y.C. Jean, Microchem. J. 42, 72 (1990).

[9] Y.Y. Wang, H. Nakanishi, J.C. Jean, T.C. Sandreczki, J. Polym. Sci. Polym. Phys. 28, 1431 (1990).

[10] Y. Kobayashi, S. Haraya, S. Hattori, T. Sasuga, Polymer 35, 927 (1994).

[11] A.J. Hill, S. Weinhold, G.M. Stack, M.R. Tant, Eur. Polym. J. 32, 843 (1996).

[12] Z.F. Wang, B. Wang, X.M. Ding, M. Zhang, L.M. Liu, N. Qi, J.L. Hu, J. Membr. Sci. 241, 355 (2004).

[13] E.-A. McGonigle, J.J. Liggat, R.A. Pethrick, S.D. Jenkins, J.H. Daly, D. Hayward, Polymer 42, 2413 (2001). 
[14] M. Misheva, N. Djourelov, E.T. Netkov, Radiation Phys. Chem. 62, 379 (2001).

[15] P. Golonka, J. Mayer, E. Dryzek, Acta Phys. Pol. A 99, 363 (2001).

[16] L. Brambilla, G. Consolati, R. Gallo, F. Quasso, F. Severini, Polymer 44, 1041 (2003).

[17] P. Dobrzyński, J. Kasperczyk, H. Janeczek, M. Bero, Macromolecules 34, 5090 (2001).

[18] O.F. Solomon, I.Z. Ciuta, J. Appl. Polym. Sci. 6, 683 (1962).

[19] J. Kansy, Nucl. Instrum. Methods Phys. Res. A 374, 235 (1996).

[20] S.J. Tao, J. Chem. Phys. 56, 5491 (1972).

[21] M. Eldrup, D. Lightbody, J.N. Sherwood, Chem. Phys. 63, 51 (1981).

[22] A. Ballara, J. Verdu, Polym. Degrad. Stab. 26, 361 (1989).

[23] N. Iliskovic, M. Bravar, Polym. Degrad. Stab. 15, 173 (1986).

[24] A. Buttafava, G. Consolati, L. Di Landro, M. Mariani, Polymer 43, 7477 (2002). 